\title{
Laser interstitial thermal therapy for palliative ablation of a chordoma metastasis to the spine: case report
}

\author{
Brian J. Williams, MD, Patrick J. Karas, MD, Ganesh Rao, MD, Laurence D. Rhines, MD, and \\ Claudio E. Tatsui, MD \\ Department of Neurosurgery, University of Texas, MD Anderson Cancer Center, Houston, Texas
}

\begin{abstract}
The authors present the first report of laser interstitial thermal therapy (LITT) ablation of a recurrent chordoma metastasis to the cervical spine. This patient was a 75-year-old woman who was diagnosed and treated for a sacral chordoma, and then developed metastases to the lung and upper thoracic spine. Unfortunately she experienced symptomatic recurrence at the C-7 spinous process. She underwent an uncomplicated LITT to the lesion. The patient convalesced without incident and was discharged on postoperative Day 1. She received stereotactic spinal radiosurgery to the lesion at a dose of $24 \mathrm{~Gy}$ in 1 fraction. At the 3-month follow-up evaluation she had radiographic response and improvement in her symptoms.

https://thejns.org/doi/abs/10.3171/2016.11.SPINE16897
\end{abstract}

KEY WORDS chordoma; metastasis; laser interstitial thermal therapy; ablation; oncology

\footnotetext{
$\mathrm{C}$
}

HORDOMA is a rare, slow-growing tumor that comprises $1 \%-4 \%$ of the primary bone malignancies. ${ }^{3}$ It has an incidence of about 1 in 1,000,000 people, has a male dominance (2-to-1 male to female ratio), and typically presents in patients between 50 and 60 years of age. ${ }^{3}$ It is hypothesized to arise from remnants of the notochord and typically occurs along the spinal axis with even distribution among the clivus, sacrum, and mobile spine. ${ }^{3}$ Chordoma cells express brachyury, a transcription factor necessary for mesoderm formation expressed within the notochord. ${ }^{6}$ Presentation varies by location but symptoms are usually nonspecific. ${ }^{2}$ Chordoma is a locally aggressive disease that uncommonly metastasizes. ${ }^{1}$ Survival is dependent primarily on radical surgical excision. If there is residual disease or recurrence then focused, high-dose radiation is the treatment of choice.? There is no approved medical treatment for chordoma, thus metastases can present a clinical dilemma. ${ }^{7}$ Given the palliative nature of treatment of chordoma metastases, open resection is not always feasible.

Laser interstitial thermal therapy (LITT) is an emerging treatment for intracranial disease. LITT utilizes frameless stereotactic guidance to precisely place a laser probe, and then intraoperative thermography to monitor treatment. LITT uses light energy to generate heat to coagulate the surrounding tissue with a sharp margin. This treatment is minimally invasive and allows for expeditious return to treatment for the primary disease. ${ }^{4}$ Furthermore, LITT has also been reported for treatment of spinal metastases in a separation surgery paradigm. ${ }^{5}$ In this paper we report the first case of recurrent metastatic chordoma treated with LITT ablation and spinal stereotactic radiosurgery (SRS).

\section{Case Report}

History and Examination

This 75-year-old woman presented with constipation and coccygeal pain, and was found to have a sacral mass. She underwent a partial sacrectomy at the S2-3 junction in January 2002. Subsequently, she was observed until 2008 when she unfortunately suffered a recurrence in her buttocks. She was treated with surgical excision and proton radiation therapy to the buttocks with a total dose of $70 \mathrm{~Gy}$, ending in August 2008. She then developed metastatic dis- 

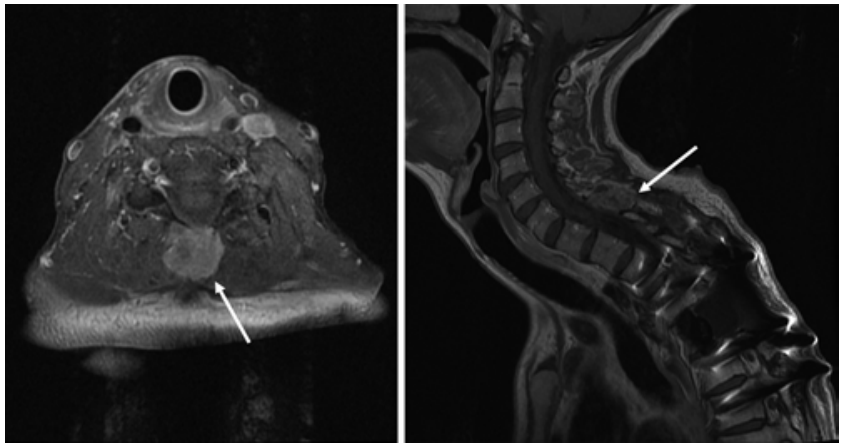

FIG. 1. Axial (left) and sagittal (right) postcontrast MR images of a C-7 spinous process lesion (arrow) representing recurrence of a metastatic chordoma deposit.

ease to T-4 with spinal cord compression in January 2010 and underwent en bloc resection of T-4 with posterior segmental stabilization from T-2 to T-6 using pedicle screws and rods. Imatinib treatment was initiated in November 2011 for slow progression of lung lesions. In June 2012, she experienced recurrence at the T-4 site and underwent revision excision of this lesion and intensity-modulated radiation therapy (IMRT) to a total dose of 50 Gy in 25 fractions from T-2 to T-6. Postoperatively, she restarted imatinib maintenance therapy.

Her most recent restaging MRI and CT scans demonstrated progression of her disease involving the spinous process of C-7 and slight progression of the paraspinal recurrent chordoma in the T3-4 region near a pedicle screw and away from the spinal canal. She was experiencing interscapular pain related to her C-7 lesion. The relationship of the T3-4 lesion to the instrumentation would limit our ability to monitor the ablation due to instrumentation artifact, thus the C-7 lesion was identified for treatment (Fig. 1).

\section{LITT and Postoperative Course}

The technique for the procedure regarding fiber placement, ablation, and damage assessment has been previously described elsewhere. ${ }^{5} \mathrm{~A}$ total of 4 laser fibers were placed with the goal of achieving complete ablation of the tumor (Fig. 2A). We placed 2 fibers from the right and 2 fibers from the left translaminar approach. Ablation was performed under breath hold. Each fiber was ablated 3 times, with each ablation lasting until either 2 minutes had elapsed, or the region of interface between the tumor and
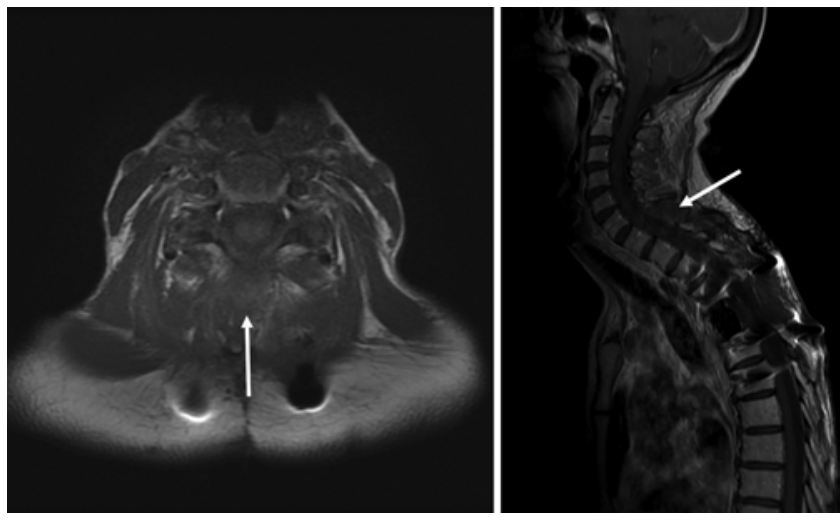

FIG. 3. Axial (left) and sagittal (right) postcontrast MR images demonstrating a durable response (arrow) at 3 months for the lesion in the C-7 spinous process.

spinal cord reached a maximum temperature of $48^{\circ}-50^{\circ} \mathrm{C}$ to prevent damage to the spinal cord. For each of the 4 fibers in this case, we achieved a temperature of $60^{\circ}-70^{\circ} \mathrm{C}$ at a distance of $7 \mathrm{~mm}$ in all directions from the tip, achieving complete coverage of the tumor. After the final ablation, we performed MRI with and without contrast enhancement and performed digital subtraction to confirm an adequate coverage of the tumor, with an area of coagulative necrosis corresponding to the preoperative contrast enhancement of the tumor (Fig. 2B and C).

The patient tolerated the procedure well and was discharged on postoperative Day 1. She underwent spinal SRS with 24 Gy in 1 fraction to the C-7 metastasis 1 week after ablation, and the T3-4 lesion was treated with 27 Gy in 3 fractions. The patient was evaluated 3 months after treatment. At that point, her symptoms had improved and her imaging demonstrated complete treatment response at C-7 (Fig. 3).

\section{Discussion}

Chordoma is a rare, locally aggressive tumor that also rarely metastasizes. There are no effective medical therapies, which limits the options for patients with metastatic chordoma. The focus of treatment for metastatic chordoma is symptom palliation. The fact that our patient had in-field failure of the thoracic tumor treated by adequate surgery and IMRT led us to believe there was a need for aggressive management of the additional lesions demonstrating radiographic progression. Yamada et al. ${ }^{8}$ reported excel-
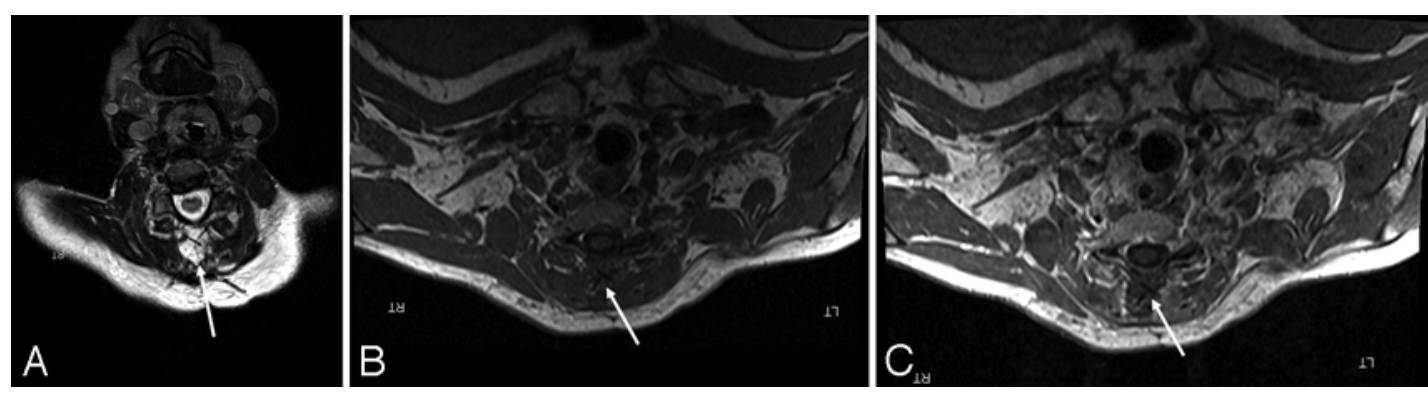

FIG. 2. Axial MR image demonstrating placement of ablation fibers into the lesion (A; arrow), and postablation axial (B and C) images with contrast demonstrating complete ablation (arrow). 
lent local control of spinal and sacral chordomas treated with single-fraction high-dose SRS, but in this series only 3 lesions were metastatic. Although encouraging, the effectiveness of SRS alone in the management of metastatic chordoma remains to be evaluated. We report the first case of using LITT ablation in association with high-dose SRS for the treatment of a metastatic chordoma to the spinous process of C-7. While pain relief is often noted after radiation treatment of chordoma metastases, we present the use of LITT as an adjunct to spinal SRS, with the goal of improving symptom control and broadening the armamentarium available in the treatment of chordoma metastases. LITT is an advantageous treatment for chordoma because it is minimally invasive with short recovery. In addition, it can be used in a complementary fashion to radiotherapy. ${ }^{5}$ Treatment of the T3-4 lesion would have been limited by instrumentation artifact, thus we elected not to attempt an ablation of this lesion. Compared with other tumor metastases types, chordomas are more lipid-rich with their myriad physaliferous bodies. While increased fat content likely decreases heat conduction, this effect was minimal as intraoperative thermography showed spherical ablation of 14-16-mm axial diameter at each fiber tip, a size comparable to other tumor types in our experience. One potential concern regarding the use of an ablative treatment such as LITT for treatment of chordoma is seeding of tumor cells along the fiber tract. Seeding may be limited by using an outer access cannula to isolate the tract between the skin and the surface of the tumor, allowing placement of the Jamshidi needle and the laser fiber without contact with the surrounding tissue.

Metastatic chordoma is a difficult disease entity to treat due to lack of medical options and limitations in radiation dosage over time. LITT is a minimally invasive modality capable of ablating soft tumoral tissue with minimal collateral damage. In this report we demonstrated, for the first time, the feasibility of using LITT in conjunction with SRS in the management of a case of metastatic chordoma.

\section{References}

1. Bjornsson J, Wold LE, Ebersold MJ, Laws ER: Chordoma of the mobile spine. A clinicopathologic analysis of 40 patients. Cancer 71:735-740, 1993
2. Cheng EY, Ozerdemoglu RA, Transfeldt EE, Thompson RC Jr: Lumbosacral chordoma. Prognostic factors and treatment. Spine (Phila Pa 1976) 24:1639-1645, 1999

3. McMaster ML, Goldstein AM, Bromley CM, Ishibe N, Parry DM: Chordoma: incidence and survival patterns in the United States, 1973-1995. Cancer Causes Control 12:1-11, 2001

4. Rahmathulla G, Recinos PF, Kamian K, Mohammadi AM, Ahluwalia MS, Barnett GH: MRI-guided laser interstitial thermal therapy in neuro-oncology: a review of its current clinical applications. Oncology 87:67-82, 2014

5. Tatsui CE, Stafford RJ, Li J, Sellin JN, Amini B, Rao G, et al: Utilization of laser interstitial thermotherapy guided by real-time thermal MRI as an alternative to separation surgery in the management of spinal metastasis. J Neurosurg Spine 23:400-411, 2015

6. Vujovic S, Henderson S, Presneau N, Odell E, Jacques TS, Tirabosco R, et al: Brachyury, a crucial regulator of notochordal development, is a novel biomarker for chordomas. $\mathbf{J}$ Pathol 209:157-165, 2006

7. Williams BJ, Raper DM, Godbout E, Bourne TD, Prevedello DM, Kassam AB, et al: Diagnosis and treatment of chordoma. J Natl Compr Canc Netw 11:726-731, 2013

8. Yamada Y, Laufer I, Cox BW, Lovelock DM, Maki RG, Zatcky JM, et al: Preliminary results of high-dose singlefraction radiotherapy for the management of chordomas of the spine and sacrum. Neurosurgery 73:673-680, 2013

\section{Disclosures}

Dr. Rhines reports being a consultant for Stryker and Globus.

\section{Author Contributions}

Conception and design: Tatsui, Williams, Karas. Acquisition of data: Tatsui. Analysis and interpretation of data: Tatsui, Williams, Karas. Drafting the article: Williams, Karas. Critically revising the article: Tatsui, Rao, Rhines. Reviewed submitted version of manuscript: Tatsui, Rao, Rhines. Approved the final version of the manuscript on behalf of all authors: Tatsui.

\section{Correspondence}

Claudio E. Tatsui, Department of Neurosurgery, University of Texas, MD Anderson Cancer Center, 1400 Holcombe Blvd., Rm. FC7.2000, Unit 442, Houston, TX 77030. email: cetatsui@ mdanderson.org. 